\title{
Operational experience with the NA62 Gigatracker
}

\author{
Massimiliano Fiorini’, S. Chiozzi, A. Cotta Ramusino, E. Gamberini, A. Gianoli, \\ F. Petrucci, H. Wahl \\ INFN Sezione di Ferrara and University of Ferrara, Italy
}

\section{G. Aglieri Rinella, D. Alvarez Feito, S. Bonacini, A. Ceccucci, J. Degrange, J. Kaplon, A. Kluge, A. Mapelli, M. Morel, J. Noël, M. Noy, L. Perktold, M. Perrin-Terrin, P. Petagna, K. Poltorak, G. Romagnoli, G. Ruggiero CERN, Geneva, Switzerland}

\section{R. Arcidiacono, C. Biino, F. Marchetto}

INFN Sezione di Torino, Italy

\section{E. Cortina Gil, E. Minucci, B. Velghe}

Université Catholique de Louvain, Belgium

The Gigatracker is a hybrid silicon pixel detector developed for the NA62 experiment at CERN, which aims at measuring the branching fraction of the ultra-rare kaon decay $\mathrm{K}^{+} \rightarrow \pi^{+} v \bar{v}$ at the CERN SPS. The detector has to track particles in a $75 \mathrm{GeV} / \mathrm{c}$ hadron beam with a flux reaching 1.3 MHz/mm² and provide single-hit timing with better than $200 \mathrm{ps}$ r.m.s. resolution for a total material budget of less than $0.5 \% \mathrm{X}_{0}$ per station. The tracker comprises three $61 \times 27 \mathrm{~mm}^{2}$ stations installed in vacuum (about $10^{-6}$ mbar) and cooled with liquid $\mathrm{C}_{6} \mathrm{~F}_{14}$ circulating through microchannels etched inside few hundred of microns thick silicon plates. Each station is composed of a $200 \mu \mathrm{m}$ thick planar silicon sensor bump-bonded to $2 \times 5$ custom $100 \mu \mathrm{m}$ thick ASIC, called TDCpix. Each chip contains $40 \times 45$ asynchronous pixels, each $300 \times 300 \mu \mathrm{m}^{2}$ and is instrumented with 720 time-to-digital converter channels with 100 ps bin. In order to cope with the high rate, the TDCpix is equipped with four $3.2 \mathrm{~Gb} / \mathrm{s}$ serializers sending out the data. Detector description, operational experience and results from the NA62 experimental runs will be presented.

The 25th International Workshop on Vertex Detectors

September 26-30, 2016

La Biodola, Isola d'Elba, ITALY

\footnotetext{
${ }^{*}$ Speaker.

${ }^{\dagger}$ Corresponding author. E-mail: fiorini@fe.infn.it
} 


\section{Introduction}

NA62 is a fixed target experiment at the CERN Super Proton Synchrotron, whose main objective is the measurement of branching fraction of the ultra-rare decay $\mathrm{K}^{+} \rightarrow \pi^{+} v \bar{v}$ with a statistical uncertainty of about $10 \%$ in a few years of data taking $[1,2]$.

The $\mathrm{K}^{+} \rightarrow \pi^{+} v \bar{v}$ is a flavour changing neutral current decays proceeding through box and electroweak penguin diagrams. A quadratic GIM mechanism and a strong Cabibbo suppression make this process extremely rare, hence extremely sensitive to physics beyond the Standard Model (SM), probing the highest mass scales among the rare meson decays. Using the value of tree-level elements of the Cabibbo-Kobayashi-Maskawa (CKM) triangle as external inputs, the SM predicts $B R\left(K^{+} \rightarrow \pi^{+} v \bar{v}\right)=(8.4 \pm 1.0) \times 10^{-11}[3,4]$. The existing measurement $B R\left(K^{+} \rightarrow \pi^{+} v \bar{v}\right)_{\exp }=$ $\left(17.3_{-10.5}^{+11.5}\right) \times 10^{-11}$ is based on 7 events (E787/E949 experiments).

The NA62 experiment plans to collect about $10^{13}$ kaon decays in few years using $400 \mathrm{GeV} / \mathrm{c}$ protons from SPS. In order to attain the required precision, a $10 \%$ signal acceptance and about $10 \%$ signal to background ratio are needed. The primary proton energy from the accelerator and the requests on kaon statistics and signal acceptance suggest the use of a kaon decay-in-flight technique similar to NA48.

The primary proton beam impinges on a beryllium target and produces plenty of secondary particles. Of those, only $75 \mathrm{GeV} / \mathrm{c}$ positively charged particles are selected in a narrow momentum band (about $1 \%)$. The resulting beam intensity is very high $(750 \mathrm{MHz})$, and the beam composition is such that only about $6 \%$ kaons are produced, the rest being pions and protons.

\section{The Gigatracker detector system}

In order to track the high intensity NA62 beam an innovative pixellated detector named Gigatracker [5] has been built, which is able to provide measurement of the momentum, time and angle of all beam tracks, and to sustain high and non-uniform particles rate. The additional requirement is that the detector should be as thin as possible in order to reduce multiple scattering and beam hadronic interactions to a minimum.

Kinematical rejection of background events require a momentum resolution of about $0.2 \%$ and angular resolution of $\sim 16 \mu \mathrm{rad}$, which can be attained with a combination of three stations at a relative distance of about ten meters, with bending magnets arranged in an "achromat" configuration, and a pixel size of $300 \times 300 \mu \mathrm{m}^{2}$. One of the most important requirements is the time resolution of better than $200 \mathrm{ps}$ on single hits, which is needed to correctly associate the parent kaon track to the daughter decay particles. In addition, a total material budget of about $0.5 \% \mathrm{X}_{0}$ has been fixed for the single station.

A prototype Gigatracker (GTK) detector has been produced and extensively studied via electrical, laser and particle beam tests. All the above requirements were successfully verified, and in particular a time resolution better than $150 \mathrm{ps}$ has been measured at high over-depletion [6]. These results led to the development of the full-scale chip for the GTK detector, called TDCpix [7, 8]

One GTK station is made of a single sensor $\left(61 \times 27 \mathrm{~mm}^{2}\right)$ bump-bonded to ten TDCpix readout chips. The GTK stations dissipate about $35 \mathrm{~W}$ each and are installed in vacuum $\left(\sim 10^{-6} \mathrm{mbar}\right)$ , therefore an efficient cooling system, and with very low material budget (about $0.15 \% \mathrm{X}_{0}$ in the 
active beam area) is necessary for stable detector operation. A micro-channel cooling solution has been adopted by the NA62 Collaboration for the first time in a high energy physics experiment [ 9 , 10]. In addition, the detector is exposed to high and non-uniform radiation levels: the expected fluence is about $2 \times 10^{14} 1 \mathrm{MeV} \mathrm{n} e q / \mathrm{cm}^{2}$ during 100 days of operation in the sensor center, and a regular exchange of detectors is performed to mitigate performance degradation due to radiation damage.

\subsection{Sensor, front-end read-out chip and micro-channel cooling plate}

Sensors used for the GTK are planar $200 \mu$ m thick p-on-n or n-on-p, produced by FBK (Trento, Italy) and CiS (Erfurt, Germany). An over-depleted operation of the detector of about $300 \mathrm{~V}$ is required to achieve the target time resolution.

Read-out wafer thinning to $100 \mu \mathrm{m}$ and flip-chip bonding to the sensor is done at IZM (Berlin, Germany), where also Benzocyclobutene (BCB) is deposited to prevent the risk of electric discharges between the sensor edges and the read-out chips.

As mentioned above, a specific read-out chip called TDCpix [7, 8] has been developed for the Gigatracker project. It is made of 40 columns of 45 pixels each for a total of 1800 pixels, for a total size of about $12 \times 20 \mathrm{~mm}^{2}$. The chip is produced in IBM $130 \mathrm{~nm}$ CMOS technology and features an on-pixel fast preamplifier and discriminator with $65 \mathrm{mV} / \mathrm{fC}$ gain, $5 \mathrm{~ns}$ peaking time and an equivalent noise charge of 170 electrons (210 with bump-bonded sensor).

The architecture is data driven, and asynchronous transmission lines transfer discriminated signal from the pixels to the end-of-column logic, where digitization takes place. Digital signals from 5 pixels in a column are sent to a "hit arbiter": a TDC pair is connected to each hit arbiter to measure leading and trailing edge times. 9 TDC pairs are used per column, for a total of 360 TDC pairs per chip that perform dual edge stamping with about 98 ps bins. Four $3.2 \mathrm{~Gb} / \mathrm{s}$ serializers per chip provide the necessary bandwidth to deal with the maximum expected $210 \mathrm{MHits} / \mathrm{s}$ rate. In addition the digital logic is triplicated in order to protect for Single Event Upsets.

The cooling plate is fabricated by CEA Leti (Grenoble, France) etching channels and openings for inlets/outlets in a $\sim 200 \mu \mathrm{m}$ thick silicon plate glued to the GTK bump-bonded assembly. Liquid coolant $\left(\mathrm{C}_{6} \mathrm{~F}_{14}\right)$ at a temperature down to $-15^{\circ} \mathrm{C}$ (at the detector inlet) is circulated in the $200 \times 70 \mu \mathrm{m}^{2}$ micro-channels at a pressure of 3.5 bars and a mass flow of $3 \mathrm{~g} / \mathrm{s}$, via two cooling circuits: fluid is brought in with capillaries and kovar connectors are soldered onto the cooling plate.

\subsection{Electro-mechanical integration}

The GTK bump-bonded assembly is glued on the micro-channel cooling plate. The cooling plate is clamped on the carrier Printed Circuit Board (PCB), then the TDCPix chips are wired bonded to the PCB (73 $\mu \mathrm{m}$ bonding pitch on chip). The carrier PCB delivers power, clock and configuration signals to the TDCpix. In addition it receives data from the TDCpix chips through 40 differential $3.2 \mathrm{~Gb} / \mathrm{s}$ lines over $30 \mathrm{~cm}$.

The carrier PCB is then glued on a frame and a flange, which closes the vacuum, allowing easy access for detector maintenance or replacement. 


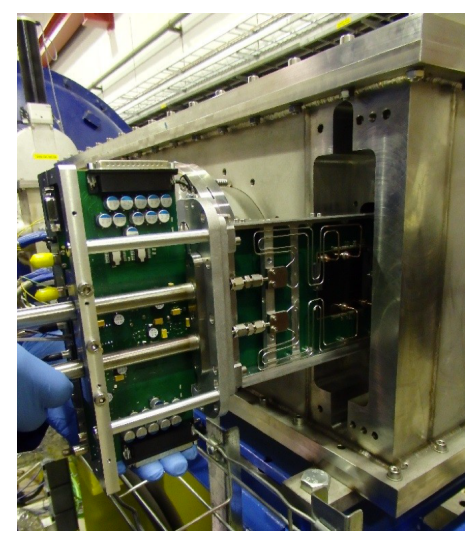

Figure 1: Installation of Gigatracker station 3 inside the vacuum vessel.

\subsection{Back-end read-out system}

As the TDCpix sends out every hit, a trigger matching needs to be implemented in the external back-end read-out system (GTK-RO), which is built using Altera Stratix GX110 FPGAs. Each GTK-RO is made of two decked cards, with a daughter card that handles the interface with Timing Trigger and Control (TTC) system of the experiment.

Each TDCpix is connected to one GTK-RO through 4 optical links (one per $3.2 \mathrm{~Gb} / \mathrm{s}$ serializer) plus 1 configuration link. The trigger matching efficiency could be measured splitting the optical signals and reading out trigger-less data concurrently (on few boards for low beam intensity).

Each GTK-RO buffers data, waiting for a level-0 trigger decision within the maximum latency of $1 \mathrm{~ms}$, retrieves data in a $75 \mathrm{~ns}$ time window upon trigger request, and finally sends data to the sub-detector PC.

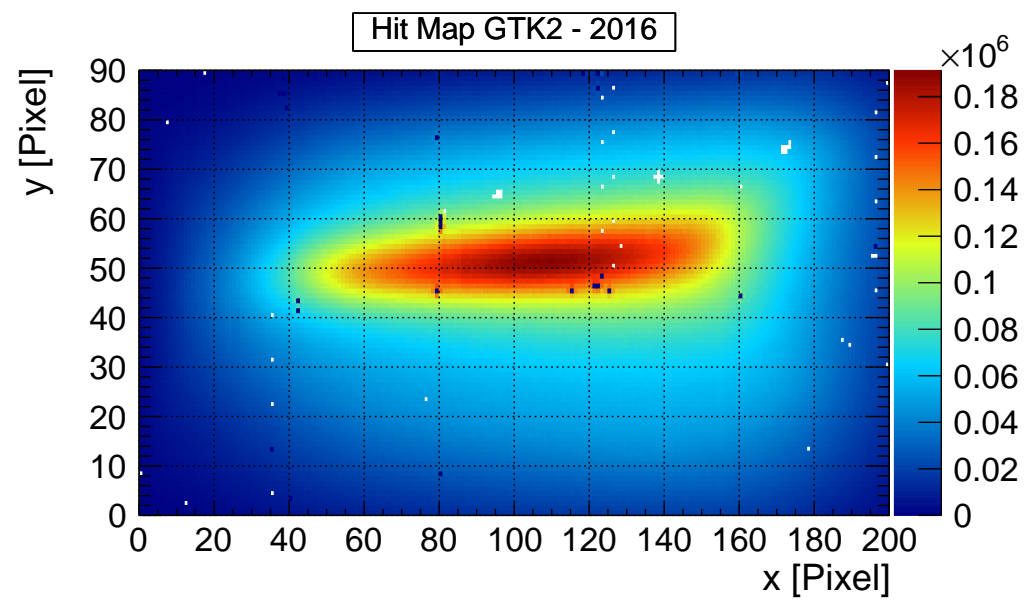

Figure 2: Particle distribution as a function of the pixel number in both horizontal $(\mathrm{X})$ and vertical $(\mathrm{Y})$ directions.

\section{Gigatracker operation and performance}

After production of single-chip and full assemblies with the final TDCpix chip, and character- 
ization in the lab with electrical and laser tests, three detector prototypes of the final design were installed in 2014 in the NA62 experimental line (Fig. 1).

The Gigatracker detector system was fully commissioned in 2015, and operated at a temperature between $-10^{\circ} \mathrm{C}$ and $0^{\circ} \mathrm{C}$, with bias voltages applied to the sensor ranging from $100 \mathrm{~V}$ to $300 \mathrm{~V}$ depending on the running periods.

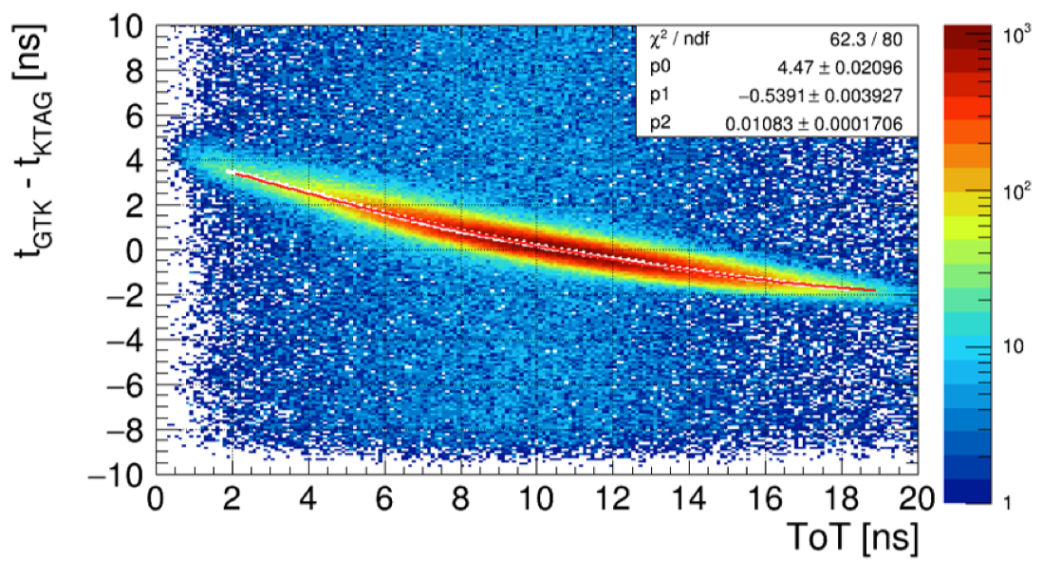

Figure 3: Plot of the time difference between GTK and KTAG as a function of the GTK time-over-threshold, from which the time-walk correction is extracted.

The NA62 beam line was commissioned up to the nominal intensity, while the sub-detectors and trigger and data acquisition systems were tested up to about $30 \%$ of the nominal beam intensity (as of September 2016).

In Fig. 2 one can observe the beam distribution at the Gigatracker station number 2. The number of non operational pixel (due either to missing bumps or noise) is at the per-mille level.

In order to correct for the time-walk effect, corrections are extracted using the Cherenkov detector called KTAG [11] as timing reference. In Fig. 3 is shown the time difference between GTK and KTAG versus the Time-over-Threshold (ToT) of the GTK hit: a fit to this distribution is done and the correction curve is then applied offline to the leading edge time to improve the time resolution.

Preliminary results on the time resolution for $300 \mathrm{~V}$ applied bias are shown in Fig. 4. The time resolution is calculated by measuring the time difference between the GTK hits and the KTAG,
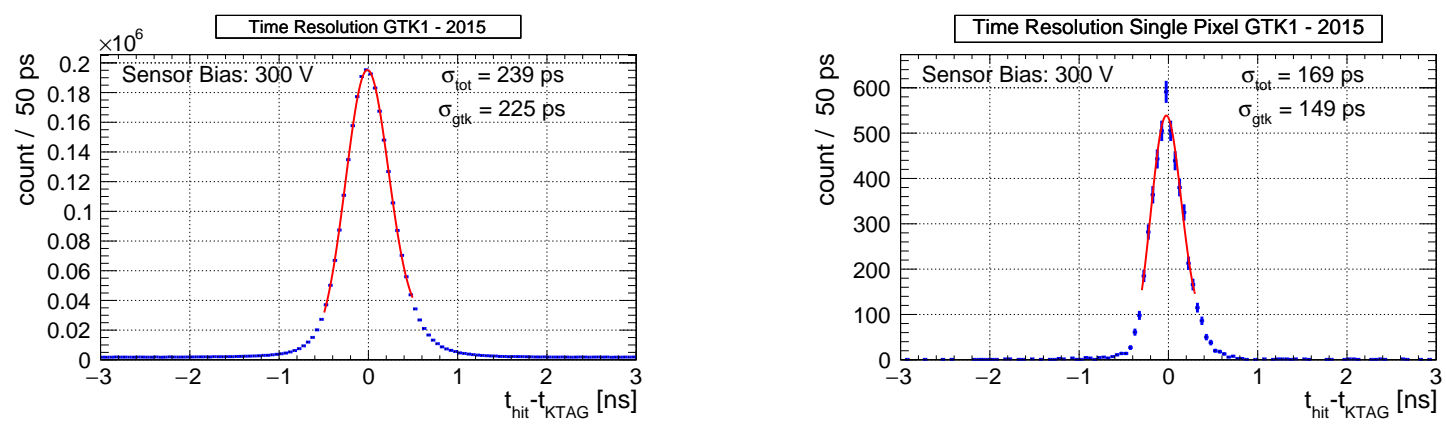

Figure 4: Time difference between the GTK and KTAG for all pixels (left) and a single pixel (right) of GTK station 1. 
and is shown on the left plot for all pixels of Gigatracker station 1. The sigma of a Gaussian fit is shown as well, together with the value calculated subtracting in quadrature the KTAG contribution. On the right plot the time resolution for a single pixel is shown: this value is compatible with the one measured during the test beam of the prototype detector, and is currently much better than the value for the whole station. The lower time resolution of the left plot is due to the fact that the time walk and time offset corrections were less precise as they had to be derived for groups of pixels to get enough statistics. Further calibrations and data analysis are needed to exploit the full potential of the detector.

The performance of the GTK detector and its contribution to the kinematics suppression of background events has been measured using data taken in special conditions with a minimum bias trigger and low beam intensity during the 2015 run. In particular, the resolution on the squared missing mass $\left(p_{K^{+}}-p_{\pi^{+}}\right)^{2}$ was measured for $K^{+} \rightarrow \pi^{+} \pi^{0}$ decays and is shown in Fig. 5 as a function of the $\pi^{+}$momentum, using information from the GTK or assuming the nominal beam momentum $(75 \mathrm{GeV} / \mathrm{c})$ travelling along the beam axis. These measurements are compared with the expected resolutions from simulations, and show the important contribution of the Gigatracker to improve the missing mass resolution by almost a factor three.

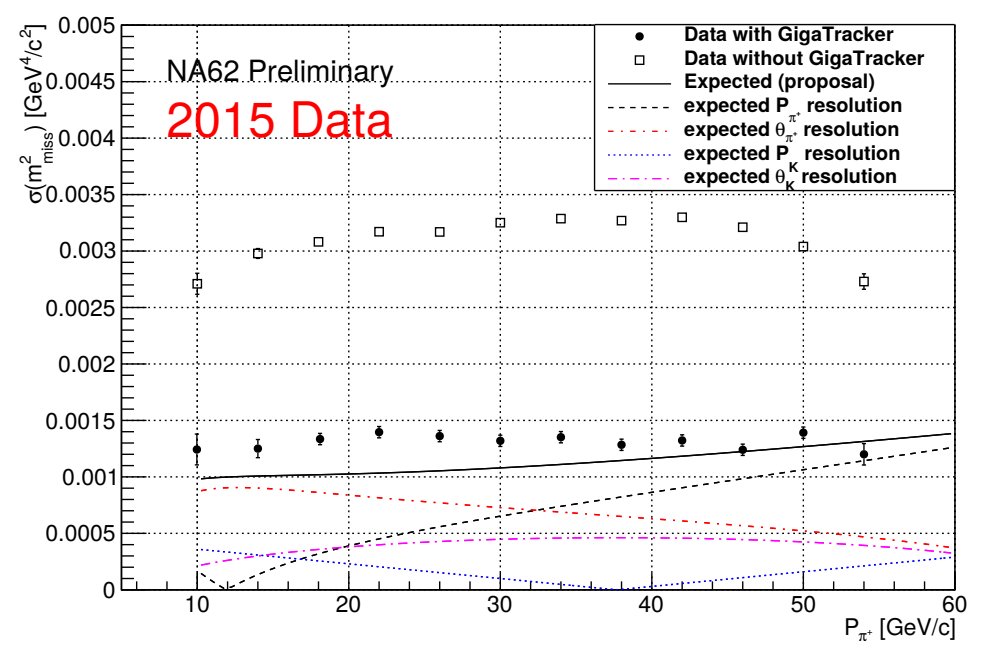

Figure 5: Squared missing mass resolution $\sigma\left(\left(p_{K^{+}}-p_{\pi^{+}}\right)^{2}\right)$ as a function of the charged pion momentum for $K^{+} \rightarrow \pi^{+} \pi^{0}$ decays, using GTK information or simply assuming the nominal beam momentum and direction [12].

\section{Conclusions}

The Gigatracker is a thin hybrid pixel detector (about $0.5 \% \mathrm{X}_{0}$ per station) with a very high density of channels (18000 per sensor), capable of measuring the NA62 beam particle tracks with a resolution better than $200 \mathrm{ps}$ in vacuum. The Gigatracker detector system has been fully commissioned in the NA62 experimental run and is taking data since 2014 showing the expected resolutions and rate capability. The Gigatracker detector allows to greatly improve kinematical rejection for 
background events, thus is an essential ingredient to precisely measure $B R\left(K^{+} \rightarrow \pi^{+} v \bar{v}\right)$. An innovative and very-low mass cooling concept based on micro-channels etched on silicon plates is being used for the first time in a high energy physics experiment and allows stable operations of the detector in vacuum. The NA62 beam intensity has been increased regularly since the start of operations and has reached its nominal value at the end of the 2016 run (November). Analysis of the full 2015 and 2016 data sets is on-going. In particular, data from the last period of the 2016 run will allow to measure the degradation of detector performance due to radiation damage.

\section{Acknowledgements}

The authors are grateful to the whole NA62 Collaboration for beam and detector operation during data taking periods, and for offline reconstruction and simulation of detector information. This work was supported by the Fonds de la Recherche Scientifique - FNRS under Grant no 4.4512.1

\section{References}

[1] G. Anelli et al., "Proposal to measure the rare decay $\mathrm{K}^{+} \rightarrow \pi^{+} v \bar{v}$ at the CERN SPS", CERN-SPSC-P-326

[2] E. Cortina Gil et al., "NA62 Technical Design Document", NA62-10-07

[3] J. Brod, M. Gorbahn and E. Stamou, "Two-Loop Electroweak Corrections for the $K \rightarrow \pi \nu \bar{v}$ Decays", Phys. Rev. D 83 (2011) 034030

[4] A. J. Buras, D. Buttazzo, J. Girrbach-Noe and R. Knegjens, " $K^{+} \rightarrow \pi^{+} v \bar{v}$ and $K_{L} \rightarrow \pi^{0} v \bar{v}$ in the Standard Model: status and perspectives”, JHEP 1511 (2015) 033

[5] M. Fiorini et al., "The P326 (NA48/3) Gigatracker: Requirements and design concept”, Nucl. Instrum. Meth. A 572 (2007) 290-291

[6] G. Aglieri Rinella et al., "Test-beam results of a silicon pixel detector with Time-over-Threshold read-out having ultra-precise time resolution”, JINST 10 (2015) no.12, P12016

[7] P. Jarron et al., "Development of the ASICs for the NA62 pixel Gigatracker", Topical Workshop on Electronics for Particle Physics, Naxos, Greece, 15 - 19 Sep 2008, pp.90-94, CERN-2008-008 (2008)

[8] A. Kluge et al., "The TDCpix readout ASIC: A 75ps resolution timing front-end for the NA62 Gigatracker hybrid pixel detector”, Nucl. Instrum. Meth. A 732 (2013) 511-514

[9] G. Nuessle, A. Mapelli, M. Morel, P. Petagna, G. Romagnoli and K. Howell, "NA62 GigaTracKer Cooling with Silicon Micro Channels", Astroparticle, Particle, Space Physics and Detectors for Physics Applications, Volume 8 (2014) 525-530

[10] G. Romagnoli et al., "Silicon micro-fluidic cooling for NA62 GTK pixel detectors", Microelectron. Eng. 145 (2015) 133

[11] E. Goudzovski et al., "Development of the kaon tagging system for the NA62 experiment at CERN" Nucl. Instrum. Meth. A 801 (2015) 86

[12] G. Ruggiero, Talk at the 51 ${ }^{s t}$ Rencontres de Moriond EW , La Thuile, Aoste, Italy, 12-19 Mar 2016 